\title{
The accuracy of reproducing target positions under various tensions
}

GEORGE E. STELMACH, University of Califormia, Santa Barbara, Calif. 93106

Using a lever positioning task, six groups of 10 Ss attempted to reproduce target positions of 25 deg or 65 deg under tension levels of either 0,5 , or $10 \mathrm{lb}$. It was found that the accuracy of reproduction was facilitated by the increased resistence at the 65 -deg target position. No increase in accuracy was found at the 25-deg target.

Proprioceptive stimuli are generally thought to perform a regulatory or feedback function for overt responses which aid in discriminating against incorrect movements. Although kinesthetic feedback is not directly accessable, some studies have attempted to manipulate the magnitude of kinesthetic feedback by adding tension to performance controls (Howland \& Noble, 1953; Weiss, 1954; Bahrick, Bennett, \& Fitts, 1955). These studies have found that increased tension on controls facilitated performance. It has been postulated by Bahrick, Fitts, \& Schnider (1955) that an increase in resistence to movement offered by a control facilitated performance by increasing the discriminability of different patterns of control movement.

In the above studies, Ss were given some form of either verbal or visual knowledge of results while discriminating against incorrect responses. In situations where no external feedback is given, the Ss must make use of the intrinsic feedback cues from the response. These kinesthetic feedback cues arise naturally from the response and are not dependent upon environmental factors. Based on the assumption that increased resistence magnifies the kinesthetic cues during a response, it is of interest to examine whether increasing the level of resistence will facilitate the accuracy of reproducing a target position. Since no feedback cues will be given, the Ss will be dependent on kinesthetic stimulation arising from the response.

\section{APPARATUS}

The apparatus consisted of a 21-in. lever mounted on the top of a table. Located 1 in. to the right of the lever arm was a $36 \times 36$ in. sheet of $3 / 4$-in. plywood upon which the target positions were defined. At the end of the lever arm was a pointer which was used to indicate the deviations of the target responses. Attached to the lever by a system of cables was a weight which exerts a force against lever displacement. A pulley and weight system was so arranged that every pound of weight at the end of the system caused $1 \mathrm{lb}$ of pull at a radial distance of $16 \mathrm{in}$. where the lever handle was located. A set of calibrated weights permitted adjustment of the dead weight to the nearest $1 / 2 \mathrm{lb}$. In the present study, the resistence of the lever arm was set at either 0,5 , or $10 \mathrm{lb}$. The resistence to lever motion was kept constant regardless of lever position by having a cable from the pulley and weight system wrap about an arc of constant radius ( 16 in.).

The $S$ sat in a hard-backed chair which was located at a right $90 \mathrm{deg}$ angle to the lever. The lever positioning response was made in the sagittal plane away from the $S$. The starting position of the lever (vertical) was defined by a permanent stop peg, and three removable stop pegs defined the target positions (25 deg and $65 \mathrm{deg}$ from vertical). $\mathrm{S}$ grasped the handle of the lever with his right hand and moved to the target position by pushing the lever in the direction $S$ was facing. PROCEDURE

The $\mathrm{S}$ was blindfolded before entering the testing room and was escorted by $\mathrm{E}$ to the apparatus. The blindfolded $\mathrm{S}$ was seated in the chair with his hand placed on the lever handle in the starting position. On the command "move," S moved the lever forward until it hit a stop peg, where he remained for 3 sec. On the command "return," $S$ moved the lever back to the starting position. Upon returning to the starting position $\mathrm{E}$ said "estimate" and S attempted to reproduce the previously defined target position with the stop peg removed. When $S$ felt he had achieved the correct position, he said, "there," and the deviations from perfect responding were recorded to the nearest $\pm 0.1 \mathrm{deg}$. The above events defined a trial and each $S$ had 10 trials under identical conditions. The intertrial interval was constant at $30 \mathrm{sec}$. All intervals were controlled by a decade interval timer.

\section{DESIGN}

Sixty male Ss were randomly assigned to six groups which made varying types of positioning responses. A 2 by 3 factorial design was used in which two target positions ( $25 \mathrm{deg}, 65 \mathrm{deg}$ ), and three tensions $(0,5,10 \mathrm{lb})$ were compared. Three groups of $10 \mathrm{Ss}$ reproduced a target position of $25 \mathrm{deg}$ with either 0 , 5 , or $10 \mathrm{lb}$ resistence; three groups of Ss reproduced a target position of $65 \mathrm{deg}$ with either 0,5 , or $10 \mathrm{lb}$ resistence.

\section{RESULTS}

Absolute errors were computed for each response and averaged across each S's 10 trials. Thus each S's score was based on 10 responses to the target position. Using these scores, the average error in degrees per movement is shown in Table 1 for each of the six groups. From inspection, it is apparent that the accuracy of reproduction is a function of the amplitudes of the movement; the errors are much larger at the 65-deg target position than the 25-deg target response. There is a definite trend of decreasing error with increasing resistence at the $65-\mathrm{deg}$ target position, while there appears to be no systematic trend at the $25-\mathrm{deg}$ response.

An analysis of variance on the means in Table 1 revealed an $F(1,54)=30.12$ for target positions which is significant $(p<.05)$. The main effect of lever tension and the Target Position by Tension interaction was nonsignificant at the .05 level.

Since the absolute error is a measure that disregards the directions of the deviations, algebraic error was also computed. For the 25-deg target response, the mean values in degrees per movement were $+1,+.5$, and +.3 for the tension levels of 0,5 , and $10 \mathrm{lb}$, respectively. At the $65-\mathrm{deg}$ target position the obtained mean values were $-.9,-.8$, and $+.3 \mathrm{deg}$. The amplitude of the movement appears to affect the direction of the deviations; Ss tend to overestimate the position of the short target and underestimate the position of the long target. It can be seen from the above mean values that there is a trend of decreasing error with increasing lever resistance only at the 65-deg target position. Analysis of the algebraic errors also revealed that the main effect of lever tension and the Target Position by Tension interaction was nonsignificant.

Although the overall $F$ was nonsignificant for the tension

Table 1

Mean Absolute Error in Degrees Per Movement for Each Tension Level at the Two Target Positions

\begin{tabular}{llcc}
\hline & \multicolumn{3}{c}{ Tension Levels } \\
\cline { 2 - 4 } & $0 \mathrm{lb}$ & $5 \mathrm{lb}$ & $10 \mathrm{lb}$ \\
\hline 25 Degree Response & 1.6 & 1.5 & 1.7 \\
65 Degree Response & 3.0 & 2.9 & 2.7 \\
\hline
\end{tabular}


levels, a closer look at the differences in tension levels was warranted due to the differential trends in the two positioning responses (Winer, 1962, p. 208). Therefore, a priori comparisons were made using the absolute error cell totals for the three tension levels at the $65-$ deg target position. The data revealed that with increasing lever tension, there was a significant decrease in error at all tension levels $(t=5.23,5.04$, and 2.57, $\mathrm{df}=54, \mathrm{p}<.05)$ indicating that increased lever resistence did facilitate the accuracy of reproduction at this target position.

\section{DISCUSSION}

The results reported here are not in agreement with the previous findings of decreasing error with increasing lever resistence in positioning responses (Bahrick, Bennett, \& Fitts, 1955; Weiss, 1954). The overall effect of tension of the two target positions was nonsignificant; however, inspection of the data revealed differential trends in the two target responses at the three tension levels. By making a priori comparisons within the 65-deg target position, it was found that the increased lever tension did significantly reduce the size of the absolute error. These findings are cautiously interpreted due to the danger of making a Type I error.

It is not clear why the $25-\mathrm{deg}$ response revealed no systematic trend with increasing lever tension, while the 65-deg response revealed decreasing error with increasing tension. This difference in the trends of the positioning responses may be attributed to the size of the errors at the two target positions. At zero tension in the 25-deg response, the absolute error was $1.6 \mathrm{deg}$ and the algebraic error was only +.1 deg. In contrast, the absolute and algebraic error was 3.0 and - .9 deg, respectively, for the $65-\mathrm{deg}$ response. The errors were sizeably larger at the $65-\mathrm{deg}$ target position allowing considerable room for improvement at this target compared to the 25-deg response.
The response in the present study was to reproduce a given target position defined by a removable stop peg. Ss were dependent solely on kinesthetic cues from moving to, and at the target position. It is possible that the errors were so small at the 25-deg response that Ss were not able to use the increased kinesthetic stimuli to discriminate against incorrect responses. Facilitation on the short responses may depend on the $S$ s receiving some kind of knowledge of results to reduce their response error, although it can be argued that increased kinesthetic stimulation at a given target position should facilitate the accuracy of reproducing that same position whether or not knowledge of results is given.

While the present study revealed that increased lever tension increased the accuracy of reproduction at only the 65-deg target position, it should be noted that the range of tension levels was small. It is possible that the increased tension levels were not of sufficient magnitude to facilitate the accuracy of reproduction when the deviations from perfect responding are small. Larger increases in tension may reveal quite different results.

\section{REFERENCES}

BAHRICK, H. P., BENNETT, W. F., \& FITTS, P. M. Accuracy of positioning responses as a function of spring loading in a control. Journal of Experimental Psychology, 1955, 49, 437-444.

BAHRICK, H. P., FITTS, P. M., \& SCHNEIDER, R. Reproduction of simple movements as a function of factors influencing proprioceptive feedback. Journal of Experimental Psychology, 1955, 49, 445-454.

HOWLAND, D., \& NOBLE, M. The effect of physical constants of a control in tracking. Journal of Experimental Psychology, 1953, 46, 353-360.

WEISS, B. The role of proprioceptive feedback in positioning responses. Journal of Experimental Psychology, 1954, 47, 215-224. NOTE

1. This study was supported by funds from Research Grant No. 571 from Research Committee, University of California, Santa Barbara. 